\title{
Postpartum brief educational interventions for women who have had gestational diabetes: a qualitative study of of health care professionals' views
}

Josie MM Evans ( $\sim$ josie.evans@stir.ac.uk)

University of Stirling

Aileen V Ireland

University of Stirling

Dawn M Cameron

University of the West of Scotland

Kate M Clarke

University of Stirling

Claire E Eades

University of Stirling

\section{Research Article}

Keywords: gestational diabetes, pregnancy, educational intervention

Posted Date: April 13th, 2021

DOI: https://doi.org/10.21203/rs.3.rs-401149/v1

License: (c) (i) This work is licensed under a Creative Commons Attribution 4.0 International License.

Read Full License 


\section{Abstract \\ Background}

Women who have had gestational diabetes during pregnancy are at very high risk of developing type 2 diabetes later in life, but their understanding of the risks is often limited. In this study we explored the views of health care professionals regarding offering brief opportunistic advice to women after their pregnancy, during unrelated consultations in primary care, relating to reducing diabetes risk.

\section{Methods}

The study took place in three Health Boards in Scotland. We conducted semi-structured one-to-one interviews (either face-to-face or telephone) with three health visitors, three practice nurses, two GPs, two diabetes consultant and two obstetricians. A focus group with five health visitors was also held. A topic guide was followed, covering the feasibility and acceptability of delivering a brief educational intervention during a routine consultation, the optimal way to identify and recall women with previous gestational diabetes, and the possible content and timing of the intervention. A thematic approach was used to analyse the qualitative data generated.

\section{Results}

The interviews/discussion lasted from 15 to 66 minutes. There was widespread support for such an intervention from all participants and general consensus that health visitors would be best placed to offer educational advice and support as part of the Health Visiting Pathway in Scotland. Thematic analysis generated three significant points of discussion: implications for training of health visitors, the need for a systematic approach to identifying women with gestational diabetes, and the optimal timing of delivery. Despite an already demanding schedule of providing advice and education to women, health visitors were confident that they could offer educational advice, provided that they received appropriate training to do so. However, there would need to be a watertight system for identifying women in their care who had had gestational diabetes. In terms of timing, later visits around 6-8 months after delivery were considered most suitable.

\section{Conclusions}

There is support from health care professionals, and most pertinently from health visitors, that the frequency of routine visits with women during the Health Visiting Pathway programme in Scotland provides potential opportunities for education around future diabetes risk to women who have had gestational diabetes. 


\section{Background}

Over $5 \%$ of all pregnancies are estimated to be complicated by gestational diabetes mellitus (GDM) ${ }^{1}$. Women who have had GDM during pregnancy are at very high risk of developing type 2 diabetes (T2D) later in life. Their risk of T2D is 10 -fold that of women who have not had GDM ${ }^{2}$. While reduced risk of T2D after GDM has been documented in women who have successfully made improvements to diet and physical activity levels post-delivery, effective interventions within heath care settings to achieve this are intensive and costly ${ }^{3}$.

We have recently completed qualitative research that has identified that there are illness perceptions surrounding GDM that, if they are not addressed, are likely to compromise the effect of lifestyle interventions ${ }^{4,5}$. For example, although many women know that there is an association between GDM and T2D, they may not perceive themselves personally as being at increased risk. For some women, GDM has minimal and transitory impact on their lives. If it is short-lived and easily controlled, they often do not appreciate the potential consequences. They may not regard T2D as particularly serious, and the lack of after-care postnatally, along with the limited mention of GDM or T2D by health care professionals, downplays its seriousness. This 'postpartum abandonment' has also been identified in other studies ${ }^{6,7}$.

There is growing interest in the use of brief opportunistic advice in the context of unrelated consultations in primary care to tackle obesity and prompt other behavioural changes ${ }^{8}$. For women with GDM, these could reduce the risk of GDM in future pregnancies, reduce their own risk of T2D and have far-reaching implications for the health and health behaviours of their offspring. Women of child-bearing age have frequent health care consultations in primary care settings. For example, the Health Visiting Pathway (HVP) in Scotland requires 11 home visits (eight in the first year of life and three between 13 months and 3-5 years $)^{9}$. We therefore conducted qualitative research with health visitors in three Health Boards in Scotland to elicit their views as to whether their routine visits could be used to deliver brief educational interventions to women who have had GDM relating to behaviour change and diabetes risk. We also interviewed two General Practitioners (GPs), three practice nurses, two consultant obstetricians and two diabetologists.

\section{Methods}

In this study we used an opt-in convenience sampling approach among health visitors, practice nurses, GPs and consultants in three Health Boards in Scotland, with populations of 300-400,000. These included a geographically large rural Health Board (A), and two Health Boards in central Scotland, each with a large urban centre (B and $C$ ).

All health care professionals were approached by email, either from lists or through personal contacts. They were sent a Participant Information Leaflet informing them of the purpose of the study, and were asked to contact the researcher if they wished to take part in an in-depth interview, or in a focus group discussion with other individuals. The researcher then followed up with telephone contact to make 
arrangements. There was only one potential participant who showed an interest in taking part, with whom we were unable to arrange a mutually convenient appointment.

Eleven interviews were carried out, either by telephone or face-to-face with the researcher, and one focus group was held with health visitors. This took place in a local Council office and was facilitated by the researcher, together with an academic nurse/midwife. The researcher and the academic nurse/midwife were both female and experienced post-doctoral qualitative researchers. Any previous contact with participants was limited to brief professional interaction.

A topic guide was used for all discussions (interviews and focus groups), and covered three main areas: the feasibility and acceptability of delivering a brief intervention during a routine consultation, the optimal way to identify and recall women with a previous GDM diagnosis, and the possible content and timing of the intervention. This semi-structured format ensured that the topics of interest were covered while allowing participants the freedom to discuss any issues not covered in the guide. Interviews lasted between 15 and 66 minutes. Written informed consent was obtained from all participants.

Discussions were audio-recorded with the participants' permission and transcribed verbatim by a professional transcription service (but not returned to the participants). A thematic approach to analysis was used ${ }^{10}$. JE and Al repeatedly read the transcripts and generated initial codes, which were then coded into sub-themes, before identifying over-arching themes.

\section{Results}

Eleven health care professionals (HCPs) were interviewed individually and five health visitors took part in a focus group discussion (Table 1). All were female. 
Table 1

Details of interviews/focus groups carried out

\begin{tabular}{|c|c|c|c|}
\hline & Health Board A & Health Board B & Health Board C \\
\hline \multirow[t]{4}{*}{ Health Visitors } & Focus group & 1 face to face interview: & 1 telephone interview: \\
\hline & 5 participants & (24 mins) & (31 mins) \\
\hline & (51 mins) & & \\
\hline & $\mathrm{p} 1$ to $\mathrm{p} 5$ & p6 & p7 \\
\hline \multirow[t]{3}{*}{ GPs } & 1 telephone & 1 face-to-face & - \\
\hline & (23 mins) & (15 mins) & \\
\hline & $\mathrm{p} 8$ & p9 & \\
\hline \multirow[t]{3}{*}{ Obstetricians } & - & 2 face-to-face interviews & - \\
\hline & & $(18,19 \mathrm{~min})$ & \\
\hline & & p10, p11 & \\
\hline \multirow[t]{3}{*}{ Diabetologists } & - & 2 face-to-face interviews & - \\
\hline & & $(16,23 \mathrm{~min})$ & \\
\hline & & $\mathrm{p} 12, \mathrm{p} 13$ & \\
\hline \multirow[t]{4}{*}{ Practice Nurses } & - & 1 face-to-face interview, & 1 telephone interview \\
\hline & & 1 telephone interview & $(28 \mathrm{~min})$ \\
\hline & & $(20,24 \mathrm{~min})$ & p16 \\
\hline & & p14, p15 & \\
\hline
\end{tabular}

There was unanimous agreement that an educational intervention for women with GDM in primary care was needed, with a typical view that 'it's just so important, it makes so much sense' (p7). This was against a backdrop of the rise in the incidence of GDM, and the 'epidemic' of diabetes (p10), meaning that the 'service was being swamped' ( $\mathrm{p} 12)$. There was recognition that some women were unaware of their increased risk of T2D or thought that it did not apply directly to them, and that many women regarded GDM as a transient diagnosis, as evidenced by the following comments:

I'm not even sure if they know they're at increased risk of diagnosis of diabetes ... it's happened when I was pregnant, it's over now, pregnancy is over, I can go back to normal now (p7)

they're not perceiving themselves to be at risk (p8)

This view was also endorsed by a diabetologist who was 'frustrated'(p11) with the situation, but recognised that postnatally 'there is a good opportunity for health promotion'( $\mathrm{p} 10)$ : 
And then they come back to you, two years on, probably heavier than they were the time before, and they're older, they need more treatment, everything is worse. So although they seem to buy into it in pregnancy, nothing happens afterwards. ( 111$)$

Despite the recognised need for an intervention, a note of caution was raised by GPs and health visitors about opportunistically offering advice in primary care. Their concerns related to the 'psychological impact of a dialogue ... about health and exercise', ensuring that this would not leave women with the over-riding sense of being 'told that they're fat'(p8). One worried 'about lowering the woman's self-esteem if we already know it's low and then we start talking about her weight'( $\mathrm{p} 1)$ and how 'it's putting pressure on them' (p1) at a challenging time. They also emphasised that the advice needed to be evidence-based.

Despite this, the central importance of behaviour change for both mother and her family was summed up well:

So it's trying to transfer the motivation that they have and the regular contact with health professionals they have during pregnancies after they've had the baby, so it's trying to engage them with that, to have that few months of really engaging them to really think, promoting the fact that this isn't just about this nine months, this is about after you've had this baby and it's for the next pregnancy and this is about ten years down the line, this is about the impact on your whole family and further generations. (p10)

The ensuing discussions were predicated that contacts generated by the health visitor pathway would be those most suited to intervention delivery, given that only two participants suggested that 'the GP is the best person to raise $i t^{\prime}(\mathrm{p} 1)$ and these two participants were health visitors themselves. All of the Practice Nurses felt that, for long-term follow up discussions, health visitors were best placed to deliver the intervention, as 'they have more contact with the women and they visit them at home' ( $\mathrm{p} 14)$. Thematic analysis generated three significant points of discussion: implications for training of health visitors, the need for a systematic approach to identifying women with GDM, and the optimal timing of delivery.

\section{Training}

There was evident concern that health visitors (and other HCPs) might need 'particular knowledge'(p13) and tailored education around GDM and its risks before they felt able to deliver a brief educational intervention, despite being potentially 'very capable' to do so (p10). One health visitor remarked: 'I think I've had my awareness raised today' ( $\mathrm{p} 4)$, with another responding 'it emphasises the need for appropriate training for the likes of $u s^{\prime}(\mathrm{p} 1)$. There was a sense that they would welcome more training in 'just knowing the questions to ask (p4) and 'just knowing the facts' (p1), and there was also the following admission:

So you can understand mothers, and some professionals, maybe thinking that is a pregnancy-related condition ( $\mathrm{p} 7$ ) 
One of the obstetricians also recalled that health visitors 'felt they didn't have the right information ... the right training to try and deliver that kind of intervention'(p12).

\section{Identification of women with GDM}

In terms of the feasibility of delivering brief educational advice, there were no systematic or watertight systems by which health visitors could be sure they knew of all women in their care who had had GDM, and this uncertainty was clearly evident: 'You would know who was gestational diabetic wouldn't you? (p5). The uncertainty is further demonstrated in the following exchange:

We've got one lady at the moment haven't we ... She's very overweight. She didn't have diabetes did she? (p2) She did (p3).

The exception was the health visitor from Health Board C, who was more confident about this, despite receiving no 'formal notification' (p7). She referred to carrying out increasing numbers of ante-natal visits where this information would be passed on, either electronically or by the woman herself, or during the handover from the midwife. However, health visitors from the other two Health Boards indicated that the information was not always received from midwifes given that this 'communication isn't as good as it used to be when we were co-located with the midwives' ( $\mathrm{p} 1$ ) and that the verbal handover from the midwife at discharge does 'not always' happen (p2). One health visitor commented, 'I've had many that have had it and when that's not been passed on' (p3). However, other explanations for this were offered. For example, GDM may be diagnosed late on in pregnancy after any handover. The relevant information 'could potentially get a little bit missed in amongst everything if it's not highlighted (p6). Finally, there was a recognition that the main focus was the baby's notes, which legally needed to be kept separate from the mother's notes.

Health visitors continue to have contact with families until the child is 5 years old. Both GPs reported that a Read code is added to the medical records of women with GDM, and that this is used for recall for blood glucose tests after delivery. While they 'presumed' that health visitors were aware of GDMcomplicated pregnancies, the systems 'would need to be a wee bit more joined up' ( $\mathrm{p} 9$ ) for continued follow-up, and for Read codes to be used to identify older children (and thus health visitors visits) whose mothers had had GDM. This is because the health records of parents are not currently linked with those of children. Overall, the proportion of mothers with GDM within one GP practice would be relatively low, so an efficient recall system would be needed. However, this could be relatively low-tech, for example, health visitors suggested having triggers (e.g. a sticker) on their paperwork, or GDM being added to the existing postnatal checklist. It was also suggested by one practice nurse that the reception team could flag up any women who missed their appointment for their 6-week postnatal GDM test (although this would not identify all women who had had GDM).

\section{Timing}

The main challenge for health visitors to be trained and deliver educational advice was perceived to be their workload, given that 'these people, these professions are so hard-pushed as it is, they don't have any 
more time to give or to work' (p10). They already have a prescriptive list of topics that need to be covered at every visit as part of the universal pathway ${ }^{9}$ for health visitors; there are always 'competing priorities' (p7), and it is also important that parents are not 'overloaded (p7). There was widespread agreement that the 6-week visit would probably be too early for delivering behaviour change advice, as the visit is so 'focused on the baby or the child' (p4), and mothers have 'so many other preoccupations with feeding the baby, lack of sleep, might have postnatal depression. Could be all sorts of things going on, so they're very much focused on just surviving day to day often' ( 1 1).

While one of the practice nurses suggested that the 12-14week health visitor check was 'a nice time because that's quite early on in the process' ( $\mathrm{p} 16)$, several health visitors mentioned the importance of the relationship that is built up with the mother, and that this kind of advice would be better delivered when 'you've got a bit of a relationship going and you hope they think you're okay and everything' (p1). Also, in the later visits, things are often more 'relaxed (p6) and by the 13-15 month visit 'women are more willing to engage about themselves rather than just their child (p5). The optional visit at 6 months or the 8month visit was deemed most appropriate for these discussions, alongside advice about weaning and food preparation for the family, but there was widespread opposition to immunisation appointments being suitable:

I wouldn't think immunisations is a good point, because parents will be really stressed actually... (p6)

\section{Discussion}

In this study we were able to elicit the views of 16 health care professionals (HCPs) from three Health Boards in Scotland, around delivering brief educational interventions to women with GDM in primary care after delivery. Although there may have been some variations in provision of care to women with GDM between the Health Boards, their views and experiences surrounding the topic seemed fairly consistent. They also chimed well with findings from other studies in a variety of settings, which suggest that many women with GDM, for various reasons, do not recognise the serious long-term implications of their GDM diagnosis $s^{3-7}$. However, we used an opt-in convenience sampling, so we cannot be sure how widespread the views of our participants were, nor whether those HCPs who opted in were those with particularly strong views or an interest in the topic. Despite these limitations, our findings respond to the recognition that a shift in perception of GDM is needed ${ }^{11}$, from one where it is assumed that it is a treatable shortterm condition, to one that has the potential to influence the long-term health of mothers and their children in the future.

There was agreement that health visitors might require more tailored training before they felt confident in delivering brief educational interventions relating to diabetes risk. This is an issue that has been recognised by other primary care researchers ${ }^{12}$ : as the incidence of GDM increases more HCPs without specialised training in diabetes will be involved de facto in the care of women who have had GDM. However, there was consensus that health visitors are well placed to delivering this kind of advice. In the context of domestic abuse, evidence suggests that health visitors may be preferred over other HCPs for 
discussing personal issues; such conversations can take place in the private spaces of women's homes and where time pressures are less problematic ${ }^{13}$. Additionally, women often report being confident in the ongoing support and guidance they receive from their health visitor, with whom they have built a relationship.

Concerns about the sensitivity of the issue could also arise, but health visitors are highly skilled professionals educated to recognise and manage sensitive issues routinely in their practice. Ensuring that any sensitive issue can be raised and addressed is based on the key health visiting principle of forming trusting relationships with women ${ }^{14}$. Health visitors are already required to screen for post-natal depression, and to raise the question of domestic abuse, undertaking a risk assessment when required.

Health visitors, as with other health professionals, juggle many competing demands. However, there needs to be consideration as to how health visitors can maximise opportunities to fit in raising this additional health concern so it is heeded and acted upon. This needs to take account of the other topics for discussion already contained within the HVP as well as ensuring the timing of the health intervention is raised before the potential seriousness of having GDM may be erased from memory. Given that many women also consider a return to work, the suggested 6-8 month period home visit would be worthy of further study as a potential opportunity for brief advice around T2D risk.

\section{Conclusions}

In summary, this study suggests that the frequency of routine visits with women during the Health Visiting Pathway (HVP) programme in Scotland provides potential opportunities to deliver brief educational interventions relating to behaviour change and diabetes risk to women who have had GDM. Despite an already demanding schedule of providing advice and education to women, the health visitors were confident that the HVP could be used to provide such interventions in the best interest of the mothers and their children. Other HCPs, those in both primary and secondary care, agreed that health visitors would be the most appropriate HCPs to for this role. It is crucial, however, that the intervention does not undermine the confidence of the women, that it is evidence-based, that health visitors are prepared with appropriate training, and that the timing of the intervention is suitable. In addition, the identification of women with GDM should be streamlined within an effective recall system to allow health visitors to adequately plan and prepare targeted interventions that correspond with routine consultations. These findings are worthy of further exploration, particularly in considering the content and timing of the educational intervention, along with the other issues identified here.

\section{Abbreviations}

GDM: gestational diabetes mellitus

T2D: type 2 diabetes

HVP: health visiting pathway 
HCP: health care professional

GP: General Practitioner

\section{Declarations}

\section{Ethics approval and consent to participate}

Informed consent was obtained from all participants. Ethical approval to conduct the study was granted from a National Health Service (NHS) Research Ethics Committee (Ref 18/LO/1955). The study was carried out in accordance with research governance policy at the University of Stirling.

\section{Consent for publication}

$\mathrm{N} / \mathrm{A}$

\section{Availability of data and materials}

Research data can be made available to interested parties upon reasonable request to the corresponding author.

\section{Competing interests}

The authors declare that they have no competing interests.

\section{Funding}

This study was funded by the Chief Scientist Office (reference CGA/18/18). The CSO was independent of the study design, the collection, analysis, and interpretation of data, and the writing of the manuscript.

\section{Author's contributions}

The project was designed by JE, DC and CE. DC and Al collected data. All authors assisted with interpretation of the data. JE wrote the first draft of the paper, and all authors contributed to the draft and approved the final content.

\section{Acknowledgements}

The authors would like to acknowledge the research participants who gave their time for this study.

\section{References}

1. Eades CE, Cameron D, Evans JMM. Prevalence of gestational diabetes mellitus in Europe: A metaanalysis. Diabetes Research and Clinical Practice 2017; 129:173-181. 
2. Vounzoulaki et al.. Progression to type 2 diabetes in women with a known history of gestational diabetes: systematic review and meta-analysis. BMJ2020;369:m1361

3. Goveia $\mathrm{P}$ et al. Lifestyle intervention for the prevention of diabetes in women with gestational diabetes mellitus: A systematic review and meta-analysis. Front Endocrino/ 2018; 5:583

4. Eades CE, Clarke K, Cameron DM, Coulson N, Evans JMM (2020). Analysis of spontaneous, usergenerated data about gestational diabetes on online forums and implications for diabetes prevention. Diabetic Med 2020;37: 2058-2066.

5. Eades CE, France E, Evans JMM. Postnatal experiences, knowledge and perceptions of women with gestational diabetes. Diabetic Med 2018; 35:519-529.

6. McMillan B, Easton K, Goyder E, Delaney B, Madhuvrata P, Abdelgalil R, Mitchell C. Reducing risk of type 2 diabetes after gestational diabetes: a qualitative study to explore the potential of technology in primary care. Br J Gen Pract 2018; 68: e260-e267

7. Parsons J, Sparrow K, Ismail K, Hunt K, Rogers H, Forbes A. A qualitative study exploring women's health behaviours after a pregnancy with gestational diabetes to inform the development of a diabetes prevention strategy. Diabetic Medicine 2019; 36: 203-213.

8. Aveyard $\mathrm{P}$, et al. Screening and brief intervention for obesity in primary care: a parallel, two-arm, randomised trial. Lancet 2016; 388: 2492-500.

9. Scottish Government (2015) Universal Health Visitor Pathway in Scotland: pre-birth to pre-school. Available: http://www.gov.scot/Resource/0048/00487884.pdf [Accessed: 3 February 21].

10. Braun V. Qualitative Research in Psychology.University of the West of England: University of the West of England; 2006. and V. Clarke, Using thematic analysis in psychology; pp. 77-101

11. Saravanan P, Magee LA, Banerjee A, Coleman MA, Von Dadelszen P, Denison F, Farmer A, Finer S, FoxRushby J, Holt R, Lindsay RS. Gestational diabetes: opportunities for improving maternal and child health. The Lancet Diabetes \& Endocrinology 2020; 8:793-800.

12. Garnweidner-Holme L, Andersen TH, Sando MW, Noll J, Lukasse M. Health care professionals' attitudes toward, and experiences of using, a culture-sensitive smartphone app for women with gestational diabetes: Qualitative study. JMIR 2018; 14.

13. Bacchus L, Bewley S, Mezey G. Experiences of seeking help from health professionals in a sample of women who experienced domestic violence. Health and Social Care in the Community 2003; 11: 1018.

14. Cowley S, Whittaker K, Grigulis A, Malone M, Donetto S, Wood H, Morrow E, Maben J. Why Health Visiting? A review of the literature about key health visitor interventions, processes and outcomes for children and families. Department of Health Policy Research Programme. 2013. London: King's College, National Nursing Research Unit. http://www.kcl.ac.uk/nursing/research/nnru/publications/index.aspx [Accessed: 15 August 20]. 